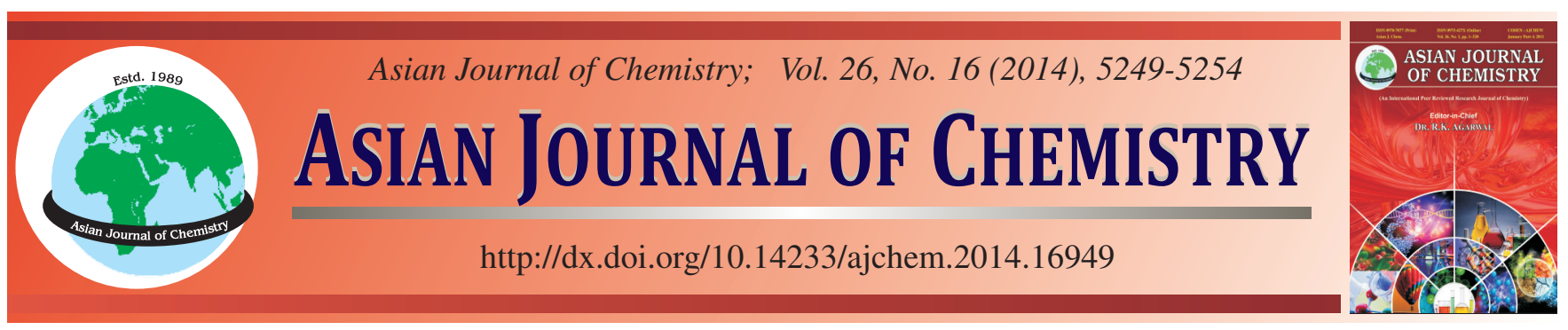

\title{
Some New NSAIDs Prodrugs: An Efficient Synthesis, Spectral Characterization and X-ray Crystal Structure Studies
}

Tarek Aboul-Fadl ${ }^{1}$, Tilal Elsaman², Omar A. Al-Deeb ${ }^{2}$, Hazem A. Ghabbour ${ }^{2}$, C.S. Chidan Kumar ${ }^{3,4}$ and Hoong-Kun Fun²,3,

${ }^{1}$ Department of Medicinal Chemistry, Faculty of Pharmacy, Assiut University, Assiut 71526, Egypt

${ }^{2}$ Department of Pharmaceutical Chemistry, College of Pharmacy, King Saud University, Riyadh 11451, Saudi Arabia

${ }^{3}$ X-ray Crystallography Unit, School of Physics, Universiti Sains Malaysia, 11800 USM, Penang, Malaysia

${ }^{4}$ Department of Chemistry, Alva's Institute of Engineering \& Technology, Mijar, Moodbidri-574 225, India

*Corresponding author: E-mail: hkfun@usm.my

\begin{abstract}
An efficient synthesis of some new NSAIDs prodrugs viz., (S)-ethyl 2-[(S)-2-(4-isobutylphenyl)propanamido]-4-methylpentanoate (1), (S)-ethyl 2-[(R)-2-(4-isobutylphenyl)propanamido]-4-methylpentanoate (2), (S)-dimethyl 2-[2-(2,3-dimethylphenylamino)benzamido]succinate (3) and (RS)-methyl-2-(2-(2,6-dichloro-3-methylphenylamino)benzamido)butanoate (4) with high purity were carried out by the amidation of RS-ibuprofen, mefenamic acid and meclofenamic acid with methyl ester and ethyl ester of amino acids L-leucine, Laspartic acid and DL-2-aminobutyric acid. The products formed were confirmed by IR, ${ }^{1} \mathrm{H}$ NMR, ${ }^{13} \mathrm{C}$ NMR, HR MS (1-4) and single crystal X-ray crystallography analysis (2-4). Compound (2) crystallizes in orthorhombic space group P212121 with unit cell dimensions $\mathrm{a}=5.1931$ (2) $\AA, \mathrm{b}=10.0031 \AA, c=41.0764$ (11) $\AA, \alpha=\beta=\gamma=90^{\circ}, \mathrm{V}=2133.80$ (12) $\AA^{3}, \mathrm{Z}=4$. Compound (3) crystallizes in hexagonal space group $\mathrm{P}_{5}$ with unit cell dimensions $\mathrm{a}=11.4965$ (3) $\AA, \mathrm{b}=11.4965$ (3) $\AA$, $\mathrm{c}=26.5323$ (12) $\AA, \alpha=\beta=90^{\circ}, \gamma=120^{\circ}, \mathrm{V}=3036.94$ (18) $\AA^{3}, Z=6$. Compound (4) crystallizes in triclinic space group Pi with unit cell dimensions a = 8.7969 (3) $\AA, \mathrm{b}=10.6307$ (3) $\AA$, c = 11.8469 (4) $\AA, \alpha=76.724(3)^{\circ}, \beta=89.066$ (3) $)^{\circ}, \gamma=66.169(2)^{\circ}, V=982.70$ (5) $\AA 3, Z=2$.
\end{abstract}

Keywords: NSAIDs, Prodrug, Conjugation, Crystal structure, Packing.

\section{INTRODUCTION}

Non-steroidal antiinflammatory drugs (NSAIDs) are a chemically diverse family of drugs that are available overthe-counter (OTC) or by prescription around the world ${ }^{1,2}$. They are widely used for the treatment of pain and chronic inflammatory ailments, such as rheumatoid arthritis ${ }^{3}$. Several epidemiological and experimental studies established NSAIDs as promising cancer chemopreventive agents ${ }^{4}$. Despite the intensive research that has been aimed at the development of NSAIDs, their clinical usefulness is still restricted by their gastrointestinal (GI) side effects, which may develop into life threatening conditions ${ }^{5}$. Ibuprofen is NSAID with anti-pyretic properties used in the therapy of rheumatism and arthritis. It is also used to treat mild to moderate pain, fever, primary dysmenorrhea and other conditions. It is available in prescription and nonprescription strengths. The racemate of various formulations is still extensively used worldwide ${ }^{6}$.

Mefenamic acid (MFA) and meclofenamic acid (MCFA), belonging to the family of $\mathrm{N}$-aryl anthranilic acid are among the widely used NSAIDs having both anti-inflammatory and analgesic activities. They suffer from general side effects of
NSAIDs, owing to the presence of free carboxylic group ${ }^{7}$. The salient features of the usefulness of conjugation of amino acids with NSAIDs have been reported ${ }^{8}$. Considerable attention has been focused on the development of bio-reversible derivatives, such as prodrugs to temporarily mask the acidic group of NSAIDs as a promising means of reducing or abolishing the gastrointestinal toxicity due to the local action mechanism. Prodrugs of NSAIDs have been prepared almost by derivatization of the carboxyl group. Esters and amides are the dominates in prodrug research, because of their ideal characteristic of exhibiting reasonable in vitro chemical stability, which allows these derivatives to be formulated with adequate shelf lives. In addition, by virtue of their ability to function as esterase substrates, esters are suitably labile, in vivo ${ }^{9,10}$. Amides and esters prodrugs of ibuprofen and fenamic acids were reported for their reduction of gastrointestinal toxicity, while maintaining their therapeutic activities as well as shifting to COX2 selectivity ${ }^{7,11}$.

Our research is aimed to develop NSAIDs prodrugs with colorectal cancer chemopreventive activity and to retard the adverse effects of gastrointestinal origin. Prodrugs of RSibuprofen, mefenamic acid and meclofenamic acid were 
synthesized by amidation with methyl and ethyl esters of amino acids L-leucine, L-aspartic acid and DL-2-aminobutyric acid.

\section{EXPERIMENTAL}

RS-Ibuprofen, mefenamic acid, meclofenamic acid and amino acid ester hydrochloride were obtained from SigmaAldrich. The other reagents and solvents used were of analytical grade. The reactions were monitored by TLC on precoated silica G plates using UV lamp. Melting points were determined on a Gallenkamp melting point apparatus and are uncorrected. Infrared Spectra were recorded as $\mathrm{KBr}$ discs using the Perkin Elmer FT-IR Spectrum BX apparatus. NMR Spectra were scanned in $\mathrm{CDCl}_{3}$ on a Bruker NMR spectrophotometer operating at $500 \mathrm{MHz}$ for ${ }^{1} \mathrm{H}$ and $125.76 \mathrm{MHz}$ for ${ }^{13} \mathrm{C}$. Chemical shifts are expressed in $\delta$-values (ppm) relative to TMS as an internal standard. Coupling constants $(\mathrm{J})$ are expressed in $\mathrm{Hz}$. $\mathrm{D}_{2} \mathrm{O}$ was added to confirm the exchangeable protons. Mass spectra were measured on Agilent Triple Quadrupole 6410 QQQ LC/MS with ESI (Electrospray ionization) source and electron impact (EI) mass fragmentations were done using Varian 320-MS, TQ Mass Spectrometer and expressed as $m / z$. Purity was confirmed using HPLC system Jasco, C18 column (Nucleosil 100/5 $\mu \mathrm{m}$ C18, 250 X $4.6 \mathrm{~mm}$, Machery Nagel and methanol: acetic acid solution $\mathrm{PH}=2.3$ (3:1) as mobile phase.

General procedure for synthesis of target prodrugs 14: The appropriate NSAID RS-ibuprofen, mefenamic acid or meclofenamic acid $(0.01 \mathrm{~mol})$ was dissolved in dichloromethane (DCM, $20 \mathrm{~mL}$ ) followed by addition of dicyclohexylcarbodiimide (DCC $0.015 \mathrm{~mol}$ ). The reaction mixture was stirred for $0.5 \mathrm{~h}$ and a mixture of amino acid hydrochloride $(0.02 \mathrm{~mol})$ and triethylamine (TEA, $0.2 \mathrm{~mL})$ in DCM $(10 \mathrm{~mL})$ was added dropwise to this mixture. The reaction mixture was stirred at $0{ }^{\circ} \mathrm{C}$ initially for $2 \mathrm{~h}$ followed by stirring at room temperature for overnight. The precipitated dicyclohexyl urea (DHU) was filtered off and discarded; the solvent was distilled off under reduced pressure. Ethyl acetate $(10 \mathrm{~mL})$ was added to the dried product to remove any remaining dicyclohexyl urea. Ethyl acetate layer was washed with aqueous solution of sodium bicarbonate $(10 \%)$ and then with distilled water to remove triethylamine hydrochloride and any traces of alkali. Ethyl acetate layer was dried over anhydrous magnesium sulphate, filtered off and the filtrate was distilled off under reduced pressure to obtain the crude product, which was then purified by column chromatography using ethyl acetate $(10 \%)$ : hexane for ibuprofen prodrug, ethyl acetate (40\%) : hexane in case of mefenamic acid and ethyl acetate $(50 \%)$ for meclofenamic acid then they were recrystallized using appropriate solvents.Data from single crystal analysis should be kept to a minimum (see Instructions for authors for further information).

Synthesis, physical and spectral characterization results of (S)-Ethyl 2-((S)-2-(4-isobutylphenyl)propanamido)-4methylpentanoate (1): Compound $\mathbf{1}$ was synthesized by the general synthetic procedure described above from reaction of RS-ibuprofen with L-leucine ethyl ester, which was isolated as white powder and crystallized from $n$-hexane (Scheme-I); Yield: $32 \%$; m.p. $59-61^{\circ} \mathrm{C}$. IR $\left(\mathrm{KBr}, \mathrm{v}_{\max }, \mathrm{cm}^{-1}\right): 3367(\mathrm{NH})$, $1713\left(\mathrm{C}=\mathrm{O}\right.$ ester), 1669 ( $\mathrm{C}=\mathrm{O}$ amide). ${ }^{1} \mathrm{H} \mathrm{NMR} ;\left(\mathrm{CDCl}_{3}\right) \delta$ $0.91\left(\mathrm{~d}, 12 \mathrm{H},\left(\mathrm{CH}_{3}\right) 2-\mathrm{CH}-\mathrm{CH}_{2} \mathrm{Ar}\right.$ and $\left.\left(\mathrm{CH}_{3}\right)_{2}-\mathrm{CH}-\mathrm{CH}_{2}-\right), 1.22-$ $1.24\left(\mathrm{~m}, 3 \mathrm{H}, \mathrm{CH}_{3}-\mathrm{CH}-\mathrm{C}=\mathrm{O}\right), 1.45\left(\mathrm{~m}, 1 \mathrm{H},-\mathrm{CH}-\left(\mathrm{CH}_{3}\right)_{2}\right), 1.54-$ $1.64\left(\mathrm{~m}, 5 \mathrm{H}, \mathrm{OCH}_{2} \mathrm{CH}_{3}\right.$ and $\left.\mathrm{CH}_{2} \mathrm{CH}\left(\mathrm{CH}_{3}\right) 2\right)$, 1.86-1.88 (m, $\left.1 \mathrm{H}, \mathrm{ArCH}_{2} \mathrm{CH}\left(\mathrm{CH}_{3}\right)_{2}\right), 2.48\left(\mathrm{~d}, 2 \mathrm{H}, J=7 \mathrm{~Hz}, \mathrm{ArCH} \mathrm{CH}_{2}\left(\mathrm{CH}_{3}\right)_{2}\right)$, 3.61 (q, $\left.J=7 \mathrm{~Hz}, 1 \mathrm{H}, \mathrm{ArCHCH}_{3}\right), 4.13$ (q, 2H, $J=7 \mathrm{~Hz}$, $\left.\mathrm{OCH}_{2}\right), 4.57$ (q, 1H, $\left.J=5 \mathrm{~Hz}, \mathrm{CHNH}\right), 5.72(\mathrm{~d}, 1 \mathrm{H}, J=6 \mathrm{~Hz}$, $\mathrm{NH}, \mathrm{D}_{2} \mathrm{O}$ exchangeable), $7.14(\mathrm{~d}, 2 \mathrm{H}, J=7 \mathrm{~Hz}, \mathrm{ArH} 3 / 5), 7.22$ $(\mathrm{d}, 2 \mathrm{H}, J=6.5 \mathrm{~Hz}, \mathrm{ArH} 2 / 6) .{ }^{13} \mathrm{C}$ NMR: $\delta 14.08\left(-\mathrm{OCH}_{2} \mathbf{C H}_{3}\right)$, $18.35\left(-\mathrm{CHCH}_{3}\right), 22.00,22.35,22.76,24.89\left(\left(\mathrm{CH}_{3}\right)_{2}-\mathrm{CH}-\right.$ $\mathrm{CH}_{2} \mathrm{Ar}$ and $\left.\left(\mathrm{CH}_{3}\right)_{2}-\mathrm{CH}-\mathrm{CH}_{2}-\right), 30.185\left(\left(\mathrm{CH}_{3}\right)_{2} \mathbf{C H C H}_{2} \mathrm{Ar}\right)$, $41.52\left(\left(\mathrm{CH}_{3}\right)_{2} \mathrm{CHCH}_{2}-\right), 45.02(\mathrm{ArCH}-), 46.67\left(\mathrm{ArCH}_{2}-\right), 50.86$ (-NHCH-), $61.17\left(\mathrm{OCH}_{2}-\mathrm{CH}_{3}\right), 127.43,129.61,138.02$, 140.79 (Ar-C), 172.78 ( $\mathrm{C}=\mathrm{O}$ amide), 174.21 ( $\mathrm{C}=\mathrm{O}$ ester). m.f. $\mathrm{C}_{21} \mathrm{H}_{33} \mathrm{NO}_{3}$, MS-ESI: $\mathrm{m} / \mathrm{z} 348.3(\mathrm{M}+1)$. MS-EI: $\mathrm{m} / \mathrm{z} 291$ (22\%), 162 (26\%), 161 (58\%), 158 (58\%), 119 (46\%), 86 $(100 \%)$.

Synthesis, physical and spectral characterization results of (S)-ethyl-2-[(R)-2-(4-isobutylphenyl)propanamido]-4methylpentanoate (2): Compound 2 was synthesized by the general synthetic procedure described above from reaction of RS-ibuprofen with L-leucine ethyl ester, which was isolated as white powder and crystallized from $n$-hexane (Scheme-I); Yield: $34 \%$; m.p. $61-63^{\circ} \mathrm{C}$. IR (KBr, $\left.v_{\max }, \mathrm{cm}^{-1}\right)$ : $3365(\mathrm{NH})$, 1751 ( $\mathrm{C}=\mathrm{O}$ ester), 1648 (C=O amide). ${ }^{1} \mathrm{H}$ NMR; $\left(\mathrm{CDCl}_{3}\right) \delta$ $0.83\left(\mathrm{~d}, 6 \mathrm{H}, J=6,\left(\mathrm{CH}_{3}\right) 2-\mathrm{CH}-\mathrm{CH}_{2}-\right), 0.91(\mathrm{~d}, 6 \mathrm{H}, J=5$ $\left.\left(\mathrm{CH}_{3}\right)_{2}-\mathrm{CH}-\mathrm{CH}_{2} \mathrm{Ar}\right), 1.26$ (t, 3H, $\left.J=7,-\mathrm{OCH}_{2} \mathrm{CH}_{3}\right), 1.38-1.6$ (m, $\left.6 \mathrm{H},\left(\mathrm{CH}_{3}\right)_{2}-\mathrm{CH}-\mathrm{CH}_{2}-\right), \mathrm{CH}_{3}-\mathrm{CH}-\mathrm{C}=\mathrm{O}$ and $\left.-\mathrm{CH}_{2} \mathrm{CH}\left(\mathrm{CH}_{3}\right)_{2}\right)$, 1.87-1.88 (m, 1H, $\left.\operatorname{ArCH}_{2} \mathrm{CH}\left(\mathrm{CH}_{3}\right)_{2}\right), 2.48(\mathrm{~d}, 2 \mathrm{H}, J=7$ $\operatorname{ArCH}{ }_{2} \mathrm{CH}\left(\mathrm{CH}_{3}\right)_{2}, 3.57\left(\mathrm{q}, J=6.5 \mathrm{~Hz}, 1 \mathrm{H}, \mathrm{ArCHCH}_{3}\right), 4.17$ (q, 2H, J = $7 \mathrm{~Hz}, \mathrm{OCH}_{2}$ ), 4.58 (q, 1H, CHNH), 5.65 (d, 1H, J $=5.5 \mathrm{~Hz}, \mathrm{NH}, \mathrm{D}_{2} \mathrm{O}$ exchangeable $), 7.14(\mathrm{~d}, 2 \mathrm{H}, J=7 \mathrm{~Hz}, \mathrm{ArH}$ 3/5), 7.21 (d, 2H, $J=7 \mathrm{~Hz}$, ArH 2/6). ${ }^{13} \mathrm{C}$ NMR: $\delta 14.1$ $\left(-\mathrm{OCH}_{2} \mathrm{CH}_{3}\right), 18.20\left(-\mathrm{CHCH}_{3}\right), 21.8,22.33,22.72,24.74$ ((CH3)2-CH- $\mathrm{CH}_{2} \mathrm{Ar}$ and $\left.\left(\mathrm{CH}_{3}\right)_{2}-\mathrm{CH}-\mathrm{CH}_{2}-\right), 30.2\left(\left(\mathrm{CH}_{3}\right)_{2-}\right.$ $\left.\left.\mathrm{CHCH}_{2} \mathrm{Ar}\right), 41.4\left(\left(\mathrm{CH}_{3}\right)_{2} \mathrm{CHCH}_{2}\right)^{-}\right), 45.0$ (ArCH-), 46.61 $\left(\mathrm{ArCH}_{2}-\right), 50.74$ (-NHCH-), $61.23\left(\mathrm{OCH}_{2}-\mathrm{CH}_{3}\right), 127.28$, 129.6, 138.51, 140.74 (Ar-C), 173.07 (C=O amide), 173.97 $\left(\mathrm{C}=\mathrm{O}\right.$ ester). m.f. $\mathrm{C}_{21} \mathrm{H}_{33} \mathrm{NO}_{3}$, MS-ESI: $m / z, 348.3(\mathrm{M}+1)$. MS-EI: $m / z 291$ (21\%), $162(27 \%), 161$ (58 \%), 158 (65\%), $119(46 \%), 86(100 \%), 57(10 \%)$.

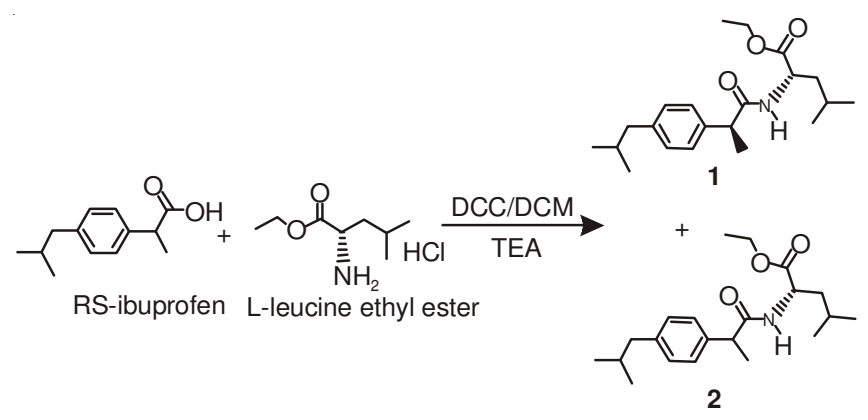

Scheme-I: Reaction of RS-ibuprofen with L-leucine ethyl ester

Similarly, mefenamic acid and meclofenamic acid were reacted with L-aspartate dimethyl ester hydrochloride and with methyl DL-2-aminobytyrate hydrochloride, respectively under the same reaction conditions mentioned in Scheme-I to afford prodrugs $\mathbf{3}$ and $\mathbf{4}$ as shown in Scheme-II. The single crystal of compound 3 was grown from $n$-hexane, while that of compound 4 was grown from ethanol. 


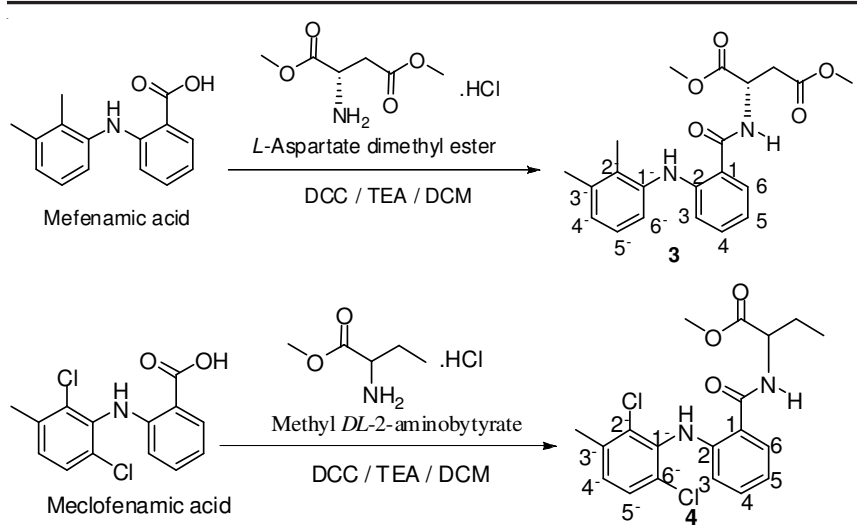

Scheme-II: Reaction of mefenamic acid and meclofenamic acid with Laspartate dimethyl ester and methyl DL-2-aminobutyrate

Synthesis, physical and spectral characterization results of (S)-dimethyl 2-[2-(2,3-dimethylphenylamino)benzamido]succinate (3): Compound 3 was synthesized by the general synthetic procedure described from reaction of mefenamic acid with L-aspartate dimethyl ester, which was isolated as yellow crystals after crystallization from $n$-hexane; Yield: $77 \%$; m.p. 82-85 ${ }^{\circ} \mathrm{C}$. IR (KBr, v, $\left.\mathrm{cm}^{-1}\right)$ : $3357(\mathrm{NH})$, 1760, 1748 ( $\mathrm{C}=\mathrm{O}$ esters), 1624 ( $\mathrm{C}=\mathrm{O}$ amide). ${ }^{1} \mathrm{H} \mathrm{NMR}$; $\left(\mathrm{CDCl}_{3}\right) \delta 2.21$ (s, 3H, $\left.\mathrm{ArC}_{2}-\mathrm{CH}_{3}\right),\left(\mathrm{s}, 3 \mathrm{H}, \mathrm{ArC}_{3}-\mathrm{CH}_{3}\right), 2.98-$ $3.02\left(\mathrm{dd}, 1 \mathrm{H}, J=4,17-\mathrm{CH}_{2} \mathrm{CO}\right), 3.15-3.20(\mathrm{dd}, 1 \mathrm{H}, J=4$, 17, $\left.-\mathrm{CH}_{2} \mathrm{CO}\right), 3.74$ (s, 3H, $\left.-\mathrm{CH}_{2} \mathrm{COOCH}_{3}\right), 3.83$ (s, 3H, $\left.\mathrm{CHCOOCH}_{3}\right), 5.07$ (t, 1H, $\left.J=4 \mathrm{~Hz},-\mathrm{CHNH}\right), 6.72(\mathrm{t}, 1 \mathrm{H}, J=$ $8 \mathrm{~Hz}, \mathrm{ArH} 5), 6.92$ (d, 1H, $J=8 \mathrm{~Hz}, \mathrm{ArH6}), 6.98$ (d, 1H, $J=7$ $\mathrm{Hz}, \mathrm{ArH} 4), 7.10$ (t, 1H, J = 7.5 Hz, Ar-H5'), 7.17 (d, 1H, $J=$ 7.5 Hz, ArH3'), 7.25-7.28 (m, 2H, Ar-H4' and -CONH which is $\mathrm{D}_{2} \mathrm{O}$ exchangeable), $7.51(\mathrm{~d}, 1 \mathrm{H}, J=7, \mathrm{ArH6}), 9.1(\mathrm{~s}, 1 \mathrm{H}$, ArNHAr, $\mathrm{D}_{2} \mathrm{O}$ exchangeable). ${ }^{13} \mathrm{C}$ NMR: $\delta 13.92\left(\mathrm{ArC}_{3}-\mathrm{CH}_{3}\right)$, $22.35\left(\mathrm{ArC}_{2}-\mathrm{CH}_{3}\right), 36.16\left(\mathrm{CH}_{2} \mathrm{CO}\right), 48.61$ (-NHCH-), 52.14 $\left(\mathrm{CH}_{2} \mathrm{COOCH}_{3}\right), 52.98\left(\mathrm{CHCOOCH}_{3}\right), 114.77\left(\mathrm{ArC}_{5}{ }^{\prime}\right), 115.5$ $\left(\mathrm{ArC}_{3}{ }^{\prime}\right), 116.7\left(\mathrm{ArC}_{4}\right), 121.32\left(\mathrm{ArC}_{1}{ }^{\prime}\right), 125.79\left(\mathrm{ArC}_{5}\right), 125.87$ $\left(\mathrm{ArC}_{6}\right), 127.81\left(\mathrm{ArC}_{6}{ }^{\prime}\right), 131.16\left(\mathrm{ArC}_{2}\right), 132.79\left(\mathrm{ArC}_{4}{ }^{\prime}\right), 138.10$ $\left(\mathrm{ArC}_{3}\right), 139.29\left(\mathrm{ArC}_{1}\right), 147.59\left(\mathrm{ArC}_{2}{ }^{\prime}\right), 169.11(\mathrm{C}=\mathrm{O}$ amide $)$, $171.37\left(\mathrm{CH}_{2} \mathrm{COOCH}_{3}\right), 171.75\left(\mathrm{CHCOOCH}_{3}\right)$. m.f. $\mathrm{C}_{21} \mathrm{H}_{24} \mathrm{~N}_{2} \mathrm{O}_{5}$, MS-ESI: $m / z 385.2\left(\mathrm{M}^{+}\right)$. MS-EI: $m / z 384.9$ (M1), 355.1 (18\%), 295 (5\%), 281 (13\%), 222 (7\%), 221 (29\%), 207 (6\%), 147 (33\%), $116(2 \%), 74(9 \%), 73(100 \%)$.

Synthesis, physical and spectral characterization results of (RS)-methyl-2-[2-(2,6-dichloro-3-methylphenylamino)benzamido]butanoate (4): Compound 4 was synthesized by the general synthetic procedure described from reaction of meclofenamic acid with methyl DL-2-aminobutyrate, which was isolated as white crystals after crystallization from ethanol; Yield: $77 \%$; m.p. $82-85{ }^{\circ} \mathrm{C}$. IR ( $\left.\mathrm{KBr}, \mathrm{v}, \mathrm{cm}^{-1}\right)$ : $3351(\mathrm{NH})$, $1732\left(\mathrm{C}=\mathrm{O}\right.$ ester), 1643 ( $\mathrm{C}=\mathrm{O}$ amide) ${ }^{1} \mathrm{H}$ NMR: $\left(\mathrm{CDCl}_{3}\right) \delta$ 0.92 (t, $\left.3 \mathrm{H}, J=7 \mathrm{~Hz},-\mathrm{CH}_{2} \mathrm{CH}_{3}\right), 1.81\left(\mathrm{~m}, 1 \mathrm{H},-\mathrm{CH}_{2} \mathrm{CH}_{3}\right), 1.97$ $\left(\mathrm{m}, 1 \mathrm{H},-\mathrm{CH}_{2} \mathrm{CH}_{3}\right), 2.32$ (s, 3H, Ar- $\left.\mathrm{CH}_{3}\right), 3.70$ (s, 3H, $-\mathrm{OCH}_{3}$ ), 4.74 (q, $1 \mathrm{H}, J=6 \mathrm{~Hz},-\mathrm{CHNH}), 6.29$ (d, 1H, $J=8 \mathrm{~Hz}, \mathrm{Ar}-\mathrm{H} 4)$, $6.90\left(\mathrm{~d}, 1 \mathrm{H}, J=6 \mathrm{~Hz}, \mathrm{CONH}-, \mathrm{D}_{2} \mathrm{O}\right.$ exchangeable), 6.72-7.46 (5H, ArH), 9.13 (s, 1H, ArNHAr, $\mathrm{D}_{2} \mathrm{O}$ exchangeable). ${ }^{13} \mathrm{C}$ NMR: $\delta 9.53\left(-\mathrm{CH}_{2} \mathbf{C H}_{3}\right), 20.66\left(\mathrm{Ar}-\mathrm{CH}_{3}\right), 25.80\left(-\mathrm{CH}_{2} \mathrm{CH}_{3}\right)$, $52.84\left(-\mathrm{OCH}_{3}\right), 53.41$ (CHNH-) 114.81, 116.92, 117.99, 127.53, 127.72, 130.22, 132.29, 133.31, 135.63, 136.73, 145.51 (ArC), 169.01 ( $\mathrm{C}=\mathrm{O}$ amide), 173.02 ( $\mathrm{C}=\mathrm{O}$ ester). m.f. $\mathrm{C}_{21} \mathrm{H}_{24} \mathrm{~N}_{2} \mathrm{O}_{5}$,
$\mathrm{C}_{19} \mathrm{H}_{20} \mathrm{~N}_{2} \mathrm{O}_{3} \mathrm{Cl}_{2}$, MS-ESI: m/z 395.2 (M+). MS-EI: m/z 387.2 (27\%), 351 (2\%), 313 (3\%), 305 (16\%), 279 (26\%), 278 (40\%), 259 (4\%), 245 (21\%), 244 (42\%), 243 (68\%) 242 (100\%), $214(16 \%), 180(20 \%), 178(12 \%), 151.1(8 \%), 92$ (5\%) $77(8 \%)$.

X-ray crystal structure analysis: X-ray crystallography studies were done for compounds 2-4 using Bruker SMART Apex II diffractometer. The data were processed with SAINT and absorption correction was done using SADABS. SHELXTL ${ }^{12}$ program was used to solve the structures of these compounds by direct method and were refined by full-matrix least squares technique on $\mathrm{F}^{2}$ using anisotropic displacement parameters. Anisotropic refinement was done for the non-hydrogen atoms. All the $\mathrm{H}$ atoms were calculated geometrically with isotropic displacement parameters set to 1.2 (1.5 for methyl groups) times the equivalent isotropic $U$ values of the parent carbon atoms. For methyl groups, a rotating group model was applied for the methyl groups. The crystallographic data and refinement parameters for the reported compounds are given in Table-1. Table-2 gives the list of $\mathrm{H}$-bonding interactions. Selected bond lengths and bond angles are given in Table- 3 and 4, respectively. CCDC 930246 for (2), 929609 for (3) and 929619 for (4) contain the supplementary crystallographic data for this paper. These data can be obtained free of charge at http://www.ccdccam.ac.uk/const/retrieving.html or from the Cambridge Crystallographic Data Centre (CCDC), 12 Union Road, Cambridge CB2 1EZ, UK; fax: +44(0)1223-336033 or e-mail: deposit@ccdc.cam.ac.uk.

\section{RESULTS AND DISCUSSION}

RS-Ibuprofen was reacted with L-Leucine ethyl ester hydrochloride in the presence of dicyclohexyl carbodiimide (DCC) and triethylamine in dichloromethane at room temperature to give the amino acid esters, prodrugs $\mathbf{1}$ and $\mathbf{2}$ as shown in Scheme-I. The latter two diastereomeric were isolatedand chromatographed in HPLC to obtain chromatograms which indicate $100 \%$ purity of $\mathbf{1}$ and $99 \%$ for $\mathbf{2}$ and so this reaction may provide an efficient way for enantiomeric resolution of racemic ibuprofen. The single crystal of compound $\mathbf{2}$ was grown from $n$-hexane using slow evaporation method, while our attempts to grow single crystal from compound $\mathbf{1}$ suitable for X-ray analyses were unsuccessful. Similarly, mefenamic acid and meclofenamic acid were reacted with L-aspartate dimethyl ester hydrochloride and with methyl DL-2-aminobutyrate hydrochloride respectively, under the same reaction conditions mentioned in Scheme-I to afford prodrugs $\mathbf{3}$ and $\mathbf{4}$ as shown in Scheme-II. The single crystal of compound $\mathbf{3}$ was grown from $n$-hexane while that of compound $\mathbf{4}$ was grown from ethanol.

X-ray crystal structure of compound 2: Colourless crystals of compound $\mathbf{2}$ were grown from $n$-hexane by slow evaporation technique. Among those, a crystal with the dimensions $0.98 \times$ $0.23 \times 0.06 \mathrm{~mm}$ was chosen and analyzed for X-ray diffraction studies. The ORTEP diagram of $\mathbf{2}$ is depicted in Fig. 1. The compound crystallizes with one unique molecule in the asymmetric unit. The aromatic ring (C11-C16) is planar with a maximum deviation of $-0.010(4) \AA$ for $\mathrm{C} 12$. The crystal packing is characterized by intermolecular $\mathrm{N} 1-\mathrm{H} 1 \mathrm{~N} \cdots \mathrm{O} 3^{\mathrm{i}}$ 


\begin{tabular}{|c|c|c|c|}
\hline \multicolumn{4}{|c|}{$\begin{array}{l}\text { TABLE-1 } \\
\text { X-RAY CRYSTAL DATA AND PARAMETERS FOR STRUCTURE REFINEMENT }\end{array}$} \\
\hline Compound & 2 & 3 & 4 \\
\hline m.f. & $\mathrm{C}_{21} \mathrm{H}_{33} \mathrm{NO}_{3}$ & $\mathrm{C}_{21} \mathrm{H}_{24} \mathrm{~N}_{2} \mathrm{O}_{5}$ & $\mathrm{C}_{19} \mathrm{H}_{20} \mathrm{Cl}_{2} \mathrm{~N}_{2} \mathrm{O}_{3}$ \\
\hline m.w. & 347.48 & 384.42 & 393.27 \\
\hline Crystal system & Orthorhombic & Hexagonal & Triclinic \\
\hline Space group & $\mathrm{P} 22_{1} 2_{1} 2_{1}$ & $\mathrm{P6}_{5}$ & $\mathrm{Pi}$ \\
\hline $\mathrm{a}\left(\AA{ }^{\circ}\right)$ & $5.1931(2)$ & $11.4965(3)$ & $8.7969(3)$ \\
\hline $\mathrm{b}(\AA)$ & 10.0031 & $11.4965(3)$ & $10.6307(3)$ \\
\hline$c(\AA)$ & $41.0764(11)$ & $26.5323(12)$ & $11.8469(4)$ \\
\hline$\alpha\left({ }^{\circ}\right)$ & 90 & 90 & $76.724(3)$ \\
\hline$\beta\left(^{\circ}\right)$ & 90 & 90 & $89.066(3)$ \\
\hline$\gamma\left({ }^{\circ}\right)$ & 90 & 120 & $66.169(2)$ \\
\hline$V\left(\AA^{3}\right)$ & $2133.80(12)$ & $3036.94(18)$ & $982.70(5)$ \\
\hline $\mathrm{Z}$ & 4 & 6 & 2 \\
\hline$D_{\text {calc }}\left(\mathrm{g} \mathrm{cm}^{-3}\right)$ & 1.082 & 1.261 & 1.336 \\
\hline$\mu\left(\mathrm{mm}^{-1}\right)$ & 0.56 & 0.75 & 3.15 \\
\hline Radiation $\lambda(\AA)$ & 1.54178 & 1.54178 & 1.54178 \\
\hline Reflections measured & 8810 & 21622 & 12846 \\
\hline Ranges/indices (h, k, l) & $-5,4 ;-12,12 ;-49,49$ & $-13,12 ;-13,13 ;-30,25$ & $-10,10 ;-12,12 ;-13,14$ \\
\hline$\theta$ limit $\left({ }^{\circ}\right)$ & $4.3-69.8$ & $4.4-69.8$ & $3.9-69.7$ \\
\hline Unique reflections & 3590 & 3385 & 3561 \\
\hline Observed reflections $(\mathrm{I}>2 \sigma(\mathrm{I}))$ & 2442 & 2369 & 2675 \\
\hline Parameters & 236 & 266 & 246 \\
\hline Goodness of fit on F2 & 1.08 & 1.02 & 1.05 \\
\hline $\mathrm{R} 1, w R 2(\mathrm{I} \geq 2 \sigma(\mathrm{I}))$ & $0.058,0.187$ & $0.053,0.158$ & $0.066,0.212$ \\
\hline
\end{tabular}

\begin{tabular}{|c|c|c|c|c|}
\hline \multicolumn{5}{|c|}{ HYDROGEN BOND GEOMETR } \\
\hline $\mathrm{D}-\mathrm{H} \cdots \mathrm{A}$ & \multicolumn{4}{|c|}{$\mathrm{d}(\mathrm{D}-\mathrm{H})(\AA)$} \\
\hline \multicolumn{2}{|l|}{$\mathrm{N} 1-\mathrm{H} 1 \mathrm{~N} \cdots \mathrm{O} 3^{\mathrm{i}}$} & \multicolumn{2}{|l|}{$0.81(4)$} & 2.4 \\
\hline \multicolumn{2}{|l|}{$\mathrm{N} 1-\mathrm{H} 1 \mathrm{~N} \cdots \mathrm{O} 1$} & \multicolumn{2}{|l|}{$0.71(4)$} & 2.1 \\
\hline \multicolumn{2}{|l|}{$\mathrm{N} 2-\mathrm{H} 2 \mathrm{~N} \cdots \mathrm{O} 1^{\mathrm{ii}}$} & \multicolumn{2}{|l|}{$0.67(4)$} & 2.3 \\
\hline \multicolumn{2}{|l|}{$\mathrm{C} 10-\mathrm{H} 10 \mathrm{~A} \cdots \mathrm{O}^{\mathrm{iii}}$} & \multicolumn{2}{|l|}{0.93} & \\
\hline \multicolumn{2}{|l|}{$\mathrm{C} 13-\mathrm{H} 13 \mathrm{~A} \cdots \mathrm{O} 1^{\mathrm{ii}}$} & \multicolumn{2}{|l|}{0.93} & \\
\hline \multicolumn{2}{|l|}{$\mathrm{C} 21-\mathrm{H} 21 \mathrm{~A} \cdots \mathrm{O} 3^{\mathrm{iv}}$} & \multicolumn{2}{|l|}{0.96} & 2.3 \\
\hline \multicolumn{2}{|l|}{$\mathrm{N} 1-\mathrm{H} 1 \mathrm{~N} 1 \cdots \mathrm{O} 1$} & \multicolumn{2}{|l|}{$0.69(4)$} & 2.1 \\
\hline \multicolumn{2}{|l|}{$\mathrm{N} 2-\mathrm{H} 2 \mathrm{~N} \cdots \mathrm{O} 2^{\mathrm{v}}$} & \multicolumn{3}{|l|}{$0.71(4)$} \\
\hline \multicolumn{2}{|c|}{$\mathrm{C} 18-\mathrm{H} 18 \mathrm{~B} \cdots \mathrm{Cg} 2^{\mathrm{vi}}$} & 0.98 & & 2.8 \\
\hline \multicolumn{5}{|c|}{$\begin{array}{l}\text { Symmetry code: (i) } x+1, y, z \text {; (ii) } x-y+1, x, z-1 / 6 \text {; (iii) } x-1, y-1, z \text {; (iv) } \\
\text { of the C7-C12 benzene ring. }\end{array}$} \\
\hline \multicolumn{4}{|c|}{$\begin{array}{c}\text { TABLE-3 } \\
\text { SELECTED BOND LENGTHS (A) } \\
\text { FOR COMPOUNDS } \mathbf{2}, \mathbf{3} \text { AND } \mathbf{4}\end{array}$} & \\
\hline \multicolumn{5}{|c|}{ Compound $\mathbf{2}$} \\
\hline $\mathrm{O} 1-\mathrm{C} 2$ & $1.474(4)$ & $\mathrm{N} 1-\mathrm{C} 4$ & $1.438(3)$ & \\
\hline $\mathrm{O} 3-\mathrm{C} 9$ & $1.228(4)$ & $\mathrm{O} 2-\mathrm{C} 3$ & $1.184(4)$ & \\
\hline $\mathrm{O} 1-\mathrm{C} 3$ & $1.331(4)$ & $\mathrm{N} 1-\mathrm{C} 9$ & $1.345(4)$ & \\
\hline \multicolumn{5}{|c|}{ Compound $\mathbf{3}$} \\
\hline $\mathrm{O} 1-\mathrm{C} 15$ & $1.244(5)$ & $\mathrm{O} 3-\mathrm{C} 20$ & $1.178(9)$ & \\
\hline $\mathrm{O} 5-\mathrm{C} 19$ & $1.443(8)$ & $\mathrm{N} 2-\mathrm{C} 15$ & $1.340(5)$ & \\
\hline $\mathrm{O} 2-\mathrm{C} 20$ & $1.283(7)$ & $\mathrm{O} 4-\mathrm{C} 18$ & $1.194(4)$ & \\
\hline $\mathrm{N} 1-\mathrm{C} 6$ & $1.410(5)$ & $\mathrm{N} 2-\mathrm{C} 16$ & $1.448(5)$ & \\
\hline $\mathrm{O} 2-\mathrm{C} 21$ & $1.56(2)$ & $\mathrm{O} 5-\mathrm{C} 18$ & $1.317(5)$ & \\
\hline $\mathrm{N} 1-\mathrm{C} 9$ & $1.365(6)$ & & & \\
\hline \multicolumn{5}{|c|}{ Compound $\mathbf{4}$} \\
\hline $\mathrm{C} 11-\mathrm{C} 1$ & $1.735(4)$ & $\mathrm{N} 1-\mathrm{C} 7$ & $1.385(4)$ & \\
\hline $\mathrm{O} 3-\mathrm{C} 16$ & $1.435(7)$ & $\mathrm{O} 2-\mathrm{C} 15$ & $1.195(5)$ & \\
\hline $\mathrm{Cl} 2-\mathrm{C} 5$ & $1.688(5)$ & $\mathrm{N} 2-\mathrm{C} 13$ & $1.353(5)$ & \\
\hline $\mathrm{N} 1-\mathrm{C} 6$ & $1.404(5)$ & $\mathrm{O} 3-\mathrm{C} 15$ & $1.333(5)$ & \\
\hline $\mathrm{O} 1-\mathrm{C} 13$ & $1.234(4)$ & $\mathrm{N} 2-\mathrm{C} 14$ & $1.440(5)$ & \\
\hline
\end{tabular}

TABLE-2

ETRIES FOR COMPOUNDS 2, 3 AND 4

\begin{tabular}{|c|c|c|c|c|}
\hline $\mathrm{D}-\mathrm{H} \cdots \mathrm{A}$ & $\mathrm{d}(\mathrm{D}-\mathrm{H})(\AA)$ & $\mathrm{d}(\mathrm{H} \cdots \mathrm{A})(\AA)$ & $\mathrm{d}(\mathrm{D} \cdots \mathrm{A})(\AA)$ & Angle $(\mathrm{D}-\mathrm{H} \cdots \mathrm{A})\left({ }^{\circ}\right)$ \\
\hline \multicolumn{5}{|c|}{ Compound $\mathbf{2}$} \\
\hline $\mathrm{N} 1-\mathrm{H} 1 \mathrm{~N} \cdots \mathrm{O} 3^{\mathrm{i}}$ & $0.81(4)$ & $2.44(4)$ & $3.226(4)$ & $166(2)$ \\
\hline \multicolumn{5}{|c|}{ Compound $\mathbf{3}$} \\
\hline $\mathrm{N} 1-\mathrm{H} 1 \mathrm{~N} \cdots \mathrm{O} 1$ & $0.71(4)$ & $2.11(4)$ & $2.675(4)$ & $137(4)$ \\
\hline $\mathrm{N} 2-\mathrm{H} 2 \mathrm{~N} \cdots \mathrm{O} 1^{\mathrm{ii}}$ & 0.67 (4) & $2.36(3)$ & $3.025(5)$ & $168(3)$ \\
\hline $\mathrm{C} 10-\mathrm{H} 10 \mathrm{~A} \cdots \mathrm{O} 5^{\mathrm{iii}}$ & 0.93 & 2.55 & $3.435(5)$ & 159 \\
\hline $\mathrm{C} 13-\mathrm{H} 13 \mathrm{~A} \cdots \mathrm{O} 1^{\mathrm{ii}}$ & 0.93 & 2.53 & $3.337(5)$ & 146 \\
\hline $\mathrm{C} 21-\mathrm{H} 21 \mathrm{~A} \cdots \mathrm{O} 3^{\text {iv }}$ & 0.96 & 2.33 & $3.126(14)$ & 140 \\
\hline \multicolumn{5}{|c|}{ Compound $\mathbf{4}$} \\
\hline $\mathrm{N} 1-\mathrm{H} 1 \mathrm{~N} 1 \cdots \mathrm{O} 1$ & $0.69(4)$ & $2.16(3)$ & $2.713(4)$ & $138(4)$ \\
\hline $\mathrm{N} 2-\mathrm{H} 2 \mathrm{~N} \cdots \mathrm{O} 2^{\mathrm{v}}$ & $0.71(4)$ & $2.39(4)$ & $3.083(4)$ & $170(5)$ \\
\hline $\mathrm{C} 18-\mathrm{H} 18 \mathrm{~B} \cdots \mathrm{Cg} 2^{\mathrm{vi}}$ & 0.98 & 2.88 & $3.742(6)$ & 149 \\
\hline
\end{tabular}

Symmetry code: (i) $x+1, y, z$; (ii) $x-y+1, x, z-1 / 6$; (iii) $x-1, y-1, z$; (iv) $y,-x+y+1, z+1 / 6$. (v) $-x,-y-1,-z+1$; (vi) $1-x,-1-y, 1-z$. $C$ g is the centroid of the $\mathrm{C} 7-\mathrm{C} 12$ benzene ring.

(Symmetry code: $\mathrm{x}+1, \mathrm{y}, \mathrm{z}$ ) hydrogen bond, connecting the molecules into chains propagating along a axis (Fig. 2 and 3). Furthermore the crystal packing is mainly stabilized by van der Waals interactions.

X-ray crystal structure of compound 3: Yellowish crystals of 3 were grown by the slow evaporation of $n$-hexane. A crystal with the dimensions $0.66 \times 0.21 \times 0.17 \mathrm{~mm}$ was selected and analyzed for X-ray diffraction studies. The molecular structure of compound $\mathbf{3}$ with atom labeling scheme is shown in Fig. 4. The aromatic ring carrying the amido group is planar with a maximum deviation of $0.008(4) \AA$ for C10. The dihedral angle between the plane of 2,3-dimethylphenyl and the benzamido group is $61.6(3)^{0}$. In the crystal structure, the $\mathrm{O} 1$ acceptor oxygen atom of the carbonyl group plays a vital role, forming an $\mathrm{N} 1-\mathrm{H} 1 \mathrm{~N}$...O 1 intramolecular hydrogen bond producing a six-membered ring with a graph-set notation of $\mathrm{S}(6)^{13}$ (Fig. 4) and intermolecular N2-H2N...O 1 and C13$\mathrm{H} 13 \mathrm{~A} \cdots \mathrm{O} 1$ bifurcated hydrogen bonds generating $\mathrm{R}_{2}{ }^{1}(7)$ ring 


\begin{tabular}{|c|c|c|c|}
\hline \multicolumn{4}{|c|}{$\begin{array}{c}\text { TABLE-4 } \\
\text { SELECTED BOND ANGLES }\left({ }^{\circ}\right) \text { FOR COMPOUNDS } 2,3 \text { AND } 4\end{array}$} \\
\hline \multicolumn{4}{|c|}{ Compound $\mathbf{2}$} \\
\hline $\mathrm{C} 2-\mathrm{O} 1-\mathrm{C} 3$ & $116.7(3)$ & $\mathrm{O} 2-\mathrm{C} 3-\mathrm{C} 4$ & $125.5(3)$ \\
\hline $\mathrm{N} 1-\mathrm{C} 4-\mathrm{C} 5$ & $111.7(3)$ & $\mathrm{O} 3-\mathrm{C} 9-\mathrm{N} 1$ & $121.6(3)$ \\
\hline $\mathrm{C} 4-\mathrm{N} 1-\mathrm{C} 9$ & $121.1(3)$ & $\mathrm{O} 1-\mathrm{C} 3-\mathrm{O} 2$ & $123.6(3)$ \\
\hline $\mathrm{N} 1-\mathrm{C} 4-\mathrm{C} 3$ & $111.0(2)$ & $\mathrm{N} 1-\mathrm{C} 9-\mathrm{C} 10$ & $115.7(3)$ \\
\hline $\mathrm{O} 1-\mathrm{C} 2-\mathrm{C} 1$ & $108.6(3)$ & $\mathrm{O} 1-\mathrm{C} 3-\mathrm{C} 4$ & $110.9(2)$ \\
\hline $\mathrm{O} 3-\mathrm{C} 9-\mathrm{C} 10$ & $122.6(3)$ & - & - \\
\hline \multicolumn{4}{|c|}{ Compound $\mathbf{3}$} \\
\hline $\mathrm{C} 20-\mathrm{O} 2-\mathrm{C} 21$ & $104.7(8)$ & $\mathrm{N} 1-\mathrm{C} 6-\mathrm{C} 1$ & $120.1(4)$ \\
\hline $\mathrm{O} 1-\mathrm{C} 15-\mathrm{C} 14$ & $123.0(3)$ & $\mathrm{O} 5-\mathrm{C} 18-\mathrm{C} 17$ & $110.4(3)$ \\
\hline $\mathrm{C} 18-\mathrm{O} 5-\mathrm{C} 19$ & $116.0(4)$ & $\mathrm{N} 1-\mathrm{C} 9-\mathrm{C} 10$ & $120.5(3)$ \\
\hline $\mathrm{N} 2-\mathrm{C} 16-\mathrm{C} 20$ & $109.7(4)$ & $\mathrm{O} 2-\mathrm{C} 20-\mathrm{C} 16$ & $112.8(6)$ \\
\hline C6-N1-C9 & $125.2(3)$ & N1-C9-C14 & $121.4(3)$ \\
\hline $\mathrm{N} 2-\mathrm{C} 16-\mathrm{C} 17$ & $111.2(3)$ & $\mathrm{O} 3-\mathrm{C} 20-\mathrm{C} 16$ & $126.3(5)$ \\
\hline $\mathrm{C} 15-\mathrm{N} 2-\mathrm{C} 16$ & $121.2(4)$ & $\mathrm{N} 2-\mathrm{C} 15-\mathrm{C} 14$ & 119.0 (4) \\
\hline $\mathrm{O} 4-\mathrm{C} 18-\mathrm{O} 5$ & $124.2(4)$ & $\mathrm{O} 2-\mathrm{C} 20-\mathrm{O} 3$ & $120.9(6)$ \\
\hline $\mathrm{N} 1-\mathrm{C} 6-\mathrm{C} 5$ & $121.0(4)$ & $\mathrm{O} 1-\mathrm{C} 15-\mathrm{N} 2$ & $118.0(3)$ \\
\hline $\mathrm{O} 4-\mathrm{C} 18-\mathrm{C} 17$ & $125.5(4)$ & - & - \\
\hline \multicolumn{4}{|c|}{ Compound 4} \\
\hline $\mathrm{C} 15-\mathrm{O} 3-\mathrm{C} 16$ & $117.2(4)$ & $\mathrm{Cl} 2-\mathrm{C} 5-\mathrm{C} 6$ & $121.5(3)$ \\
\hline $\mathrm{N} 1-\mathrm{C} 7-\mathrm{C} 12$ & $120.0(3)$ & $\mathrm{N} 2-\mathrm{C} 14-\mathrm{C} 17$ & $110.4(3)$ \\
\hline $\mathrm{C} 6-\mathrm{N} 1-\mathrm{C} 7$ & $124.9(3)$ & $\mathrm{Cl} 2-\mathrm{C} 5-\mathrm{C} 4$ & $120.0(4)$ \\
\hline $\mathrm{O} 1-\mathrm{C} 13-\mathrm{C} 12$ & $122.9(3)$ & $\mathrm{O} 2-\mathrm{C} 15-\mathrm{C} 14$ & $125.1(4)$ \\
\hline $\mathrm{C} 13-\mathrm{N} 2-\mathrm{C} 14$ & $120.0(3)$ & $\mathrm{N} 1-\mathrm{C} 6-\mathrm{C} 1$ & $121.4(3)$ \\
\hline $\mathrm{N} 2-\mathrm{C} 13-\mathrm{C} 12$ & $117.3(3)$ & $\mathrm{O} 3-\mathrm{C} 15-\mathrm{C} 14$ & $110.6(3)$ \\
\hline $\mathrm{C} 11-\mathrm{C} 1-\mathrm{C} 6$ & $117.6(3)$ & $\mathrm{N} 1-\mathrm{C} 6-\mathrm{C} 5$ & $120.9(3)$ \\
\hline $\mathrm{O} 1-\mathrm{C} 13-\mathrm{N} 2$ & $119.8(3)$ & $\mathrm{O} 2-\mathrm{C} 15-\mathrm{O} 3$ & $124.3(4)$ \\
\hline $\mathrm{C} 11-\mathrm{C} 1-\mathrm{C} 2$ & $118.4(3)$ & $\mathrm{N} 1-\mathrm{C} 7-\mathrm{C} 8$ & 120.7 (3) \\
\hline $\mathrm{N} 2-\mathrm{C} 14-\mathrm{C} 15$ & $110.9(3)$ & - & - \\
\hline
\end{tabular}

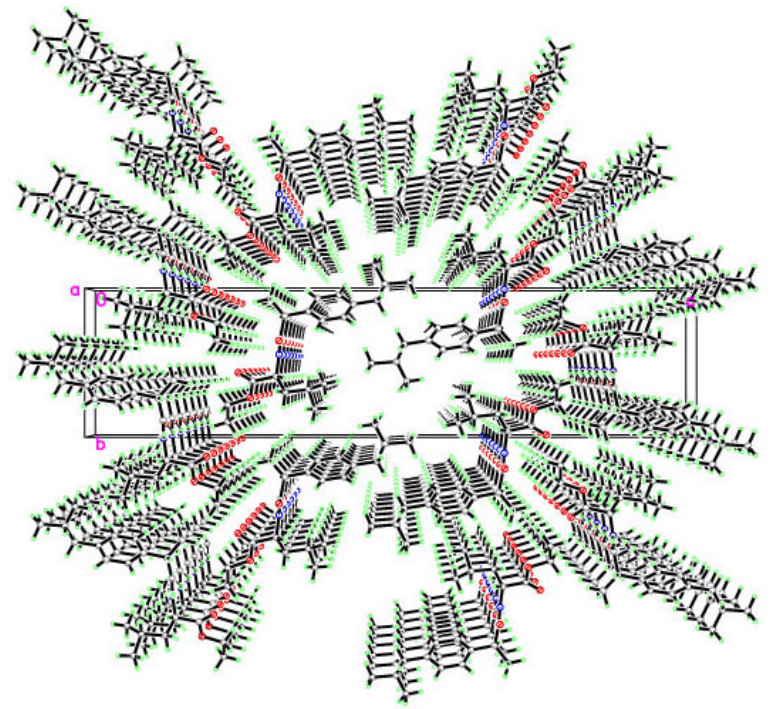

Fig. 3. Packing diagram of $\mathbf{2}$ viewed down the a axis

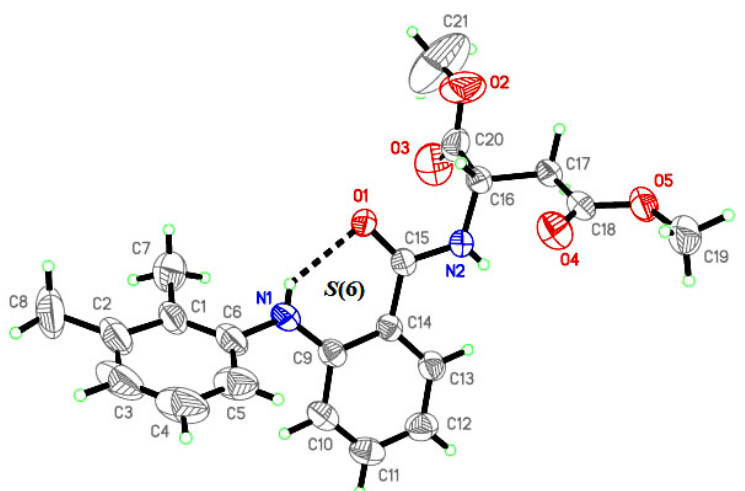

Fig. 4. Molecular structure of compound $\mathbf{3}$ with atom labeling scheme

motif. The crystal structure is further stabilized by $\mathrm{C} 10$ -

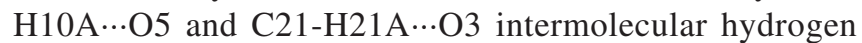
bonds forming a three dimensional network as shown in Fig. 5.

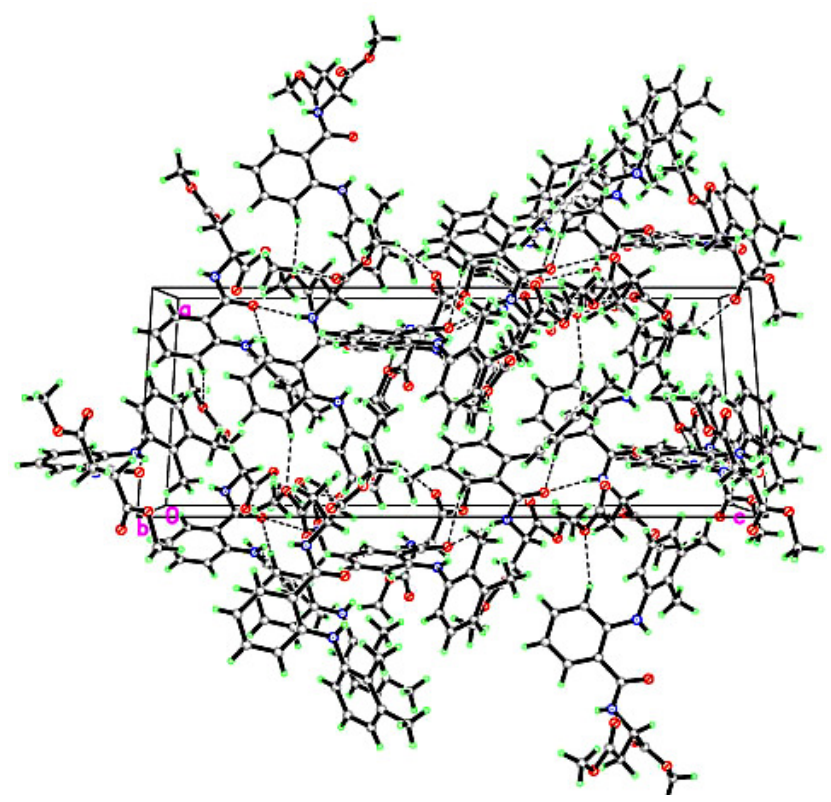

Fig. 5. Molecules are linked to form a three-dimensional network. Dashed lines indicate the intermolecular $\mathrm{H}$-bonds 
X-ray crystal structure of compound 4: Colourless needle-shaped crystals of compound $\mathbf{4}$ were grown from ethanol by slow evaporation technique. A crystal with the dimensions $0.31 \times 0.13 \times 0.10 \mathrm{~mm}$ was selected and analyzed for X-ray diffraction studies. The molecular structure of compound $\mathbf{4}$ drawn at $50 \%$ ellipsoids for non-hydrogen atoms is shown in Fig. 6. The benzamido ring (C7-C12) is planar with a maximum deviation of -0.008(4) $\AA$ for $\mathrm{C} 12$ and forms an dihedral angle of $69.0(2)^{0}$ to the 2,6-dichloro-3-methylphenyl ring (C1-C6). The torsion angle of 71.7(5) for C7-N1-C6-C5 indicates the deviation of 2,6-dichloro-3-methylphenyl ring system from that of the plane of benzamido ring system. Similar to compound 3 (Fig. 4), in the molecular structure of $\mathbf{4}$, an intramolecular N1-H1N1 ‥O1 hydrogen bond with an S(6) graphset ring motif ${ }^{13}$ is observed (Fig. 6). A pair of intermolecular N2-H2N … 2 (Symmetry code: $-\mathrm{x},-\mathrm{y}-1,-\mathrm{z}+1$ ) hydrogen bonds link the molecules to form inversion dimer, producing the $\mathrm{R}_{2}^{2}(10)$ ring motif. The crystal structure also features for $\mathrm{Cl} \cdots \mathrm{Cl}$ (halogen-halogen) interactions, which is shown in Fig. 7. Further, the crystal packing stability is consolidated by weak $\pi-\pi$ (centroid-centroid distance $=3.722(2) \AA$ ) and $\mathrm{C}-\mathrm{H} \cdots \mathrm{p}$ interactions.

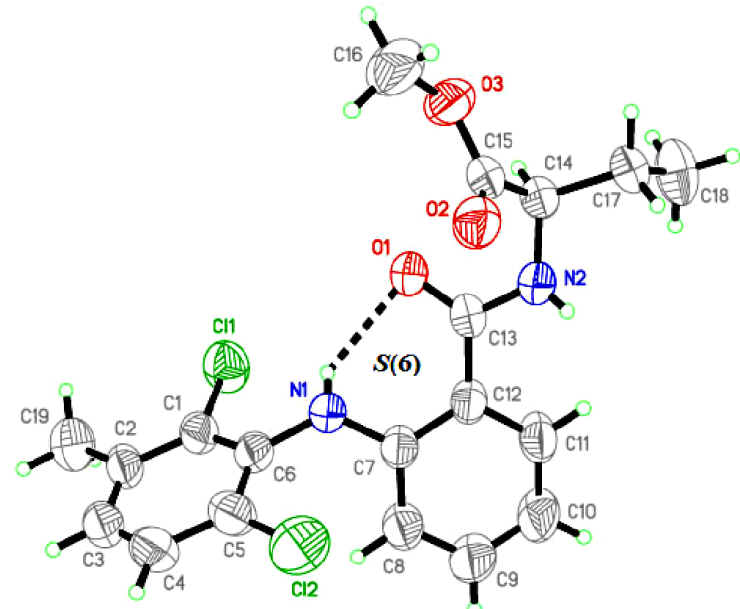

Fig. 6. ORTEP diagram of compound $\mathbf{4}$ drawn at $50 \%$ ellipsoids for nonhydrogen atoms

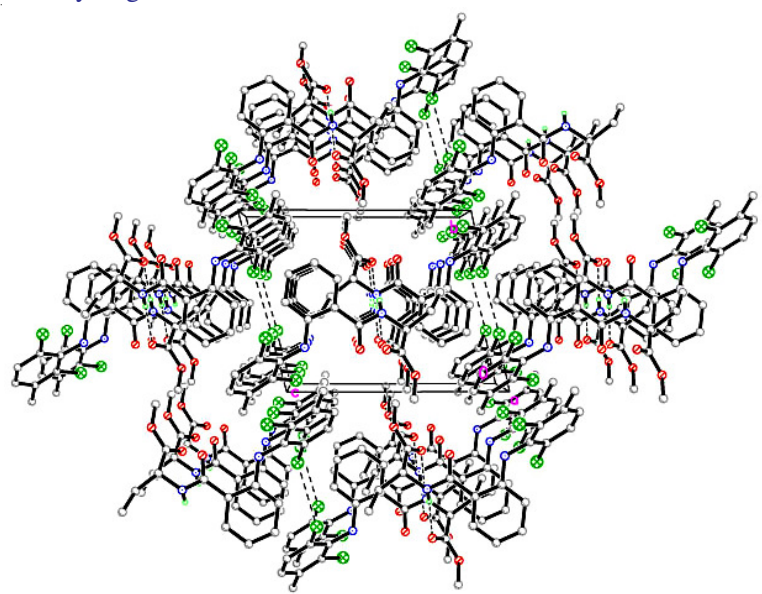

Fig. 7. Crystal packing of (4) showing intermolecular N-H $\cdots \mathrm{O}$ hydrogen bonding and $\mathrm{Cl} \cdots \mathrm{Cl}$ (halogen-halogen) interactions as dashed lines. $\mathrm{H}$ atoms not involved in the $\mathrm{H}$-bonding have been omitted for clarity

\section{Conclusion}

Some new NSAIDs prodrugs were efficiently synthesized by amidation of RS-ibuprofen, mefenamic acid and meclofenamic acid with methyl and ethyl esters of amino acids Lleucine, L-aspartic acid and DL-2-aminobutyric acid. This method yielded the products with high purity. The structural characteristics of these prodrugs were confirmed by IR, ${ }^{1} \mathrm{H}$ NMR, ${ }^{13} \mathrm{C}$ NMR, HR MS (1-4) and by single crystal X-ray crystallography analysis (2-4). The molecular structures and significance of $\mathrm{H}$-bond patterns consolidating the crystal stability in these compounds are discussed.

\section{ACKNOWLEDGEMENTS}

The authors are thankful to King Abdul-Aziz City for Science and Technology for funding the work by research grant No. A-S-12-0893 and the Deanship of Scientific Research and the Research Center, College of Pharmacy, King Saud University. One of the authors, C.S. Chidan Kumar thanks Universiti Sains Malaysia for a postdoctoral research fellowship.

\section{REFERENCES}

1. F. Bessone, World J. Gastroenterol., 16, 5651 (2010).

2. H.E. Vonkeman and M.A. van de Laar, Semin. Arthritis Rheum., 39, 294 (2010).

3. J. Steinmeyer, Arthritis Res., 2, 379 (2000).

4. T. Aboul-Fadl, S.S. Al-Hamad, M.K. Abdel-Hamid, H.A. Abdel-Aziz, A.M. Al-Obaid, J.D. Whitt, B.D. Gary, A.B. Keeton and G.A. Piazza, 243rd American Chemical Society Meeting, ACS, San Diego, California, USA, March (2012).

5. R.T. Schoen and R.J. Vender, Am. J. Med., 86, 449 (1989),

6. H. Hao, G. Wang and J. Sun, Drug Metab. Rev., 37, 215 (2005).

7. A. Rasheed and C.K. Ashok-Kumar, Int. J. Curr. Pharm. Res., 1, 47 (2009).

8. T. Aboul-Fadl and E.A. Fouad, Pharmazie, 51, 30 (1996).

9. H. Bundgaard, Adv. Drug Deliv. Rev., 3, 39 (1989).

10. H. Bundgaard, In Design of Prodrugs, Elsevier, New York, Plenum Press (1986).

11. E. Banoglu, B. Okcelik, E. Kupeli, S. Unlu, E. Yesilada, M. Amat, J.F. Caturla and M.F. Sahin, Arch. Pharm., 336, 251 (2003).

12. G.M. Sheldrick, Acta Crystallogr. A, 64, 112 (2008)

13. J. Bernstein, R.E. Davis, L. Shimoni and N.L. Chang, Angew. Chem. Int. Ed. Engl., 34, 1555 (1995). 